\title{
Ausgewählte Aspekte und erste Erfahrungen bei der Vor- bereitung, Planung und Realisierung von KMU-Netzwerken
}

\author{
Dr.-Ing. Helmut Bärthel, Prof. Dr. sc. oec. Michael Büttner
}

\section{Einführung}

Die im Rahmen dieses Beitrages zur Vorbereitung, Planung und Realisierung von Unternehmensnetzwerken betrachteten Aspekte, Ursachen, Bedingungen und Erfahrungen aus der Evaluierung eines extrem komplizierten, strategisch angelegten Strukturierungsprozesses im Bereich der kleinen und mittleren Unternehmen (KMU) fundieren primär auf drei Quellen. Erste Quelle ist eine Studie des Institutes für Strukturpolitik und Wirtschaftsförderung Halle-Leipzig (isw) zu „Netzwerken in Sachsen-Anhalt" [1], die Quellen [2] und [3] sind Forschungsberichte zu den Themen „Aufbau eines Netzwerkes zur Verwirklichung des ökologisch-ökonomischen Bauens unter Beteiligung von kleinen und mittleren Unternehmen verschiedener Branchen“ in der Region Halle-Dessau-Bitterfeld-Wolfen [2] und „Aufbau eines absatzorientierten Netzwerkes kleiner und mittlerer Unternehmen im Raum Leuna-Merseburg"- auch als Chemiedreieck bezeichnet [3]. Die beiden Netzwerkprojekte wurden vom isw initiiert und planungsseitig vorbereitet, werden vom isw wissenschaftlich begleitet und vom Land Sachsen-Anhalt gefördert.

\section{Definition und Diskussion zum Begriff Netzwerk}

Unter Verzicht auf eine Auseinandersetzung mit den verschiedenen Definitionen für den Begriff "Netzwerk“ (vgl.dazu [7], [8]) soll im Rahmen der vorliegenden Arbeit eine Definition von Sydow [4] zugrunde gelegt werden. Er schreibt: „Ein Unternehmensnetzwerk stellt eine auf die Realisierung von Wettbewerbsvorteilen zielende Organisationsform ökonomischer Aktivitäten dar, die sich durch komplex-reziproke eher kooperative denn kompetive und relativ stabile Beziehungen zwischen rechtlich selbständigen, wirtschaftlich jedoch zumeist abhängigen Unternehmen auszeichnet.

Ein derartiges Netzwerk, das entweder in einer oder in mehreren miteinander verflochtenen Branchen agiert, ist das Ergebnis einer Unternehmensgrenzen übergreifenden Differenzierung und Integration ökonomischer Aktivitäten."

Er ergänzt: „Ein Unternehmensnetzwerk besteht folglich aus einer endlichen Anzahl von rechtlich und - in einem noch eingeschränkten Sinne als die Unternehmung wirtschaftlich selbständigen Einheiten. Diese werden als Netzwerkunternehmungen bezeichnet."

Die erkennbaren Festpunkte in dieser Definition sind:

- Unternehmensnetzwerk, d.h., es gibt eine definierte Menge von Unternehmen oder Teilen davon, die sich zum Zwecke der Erarbeitung gemeinsamer Produkte oder Leistungen für einen bestimmten Zeitraum „zusammentun“, man spricht auch vom „Bündeln der Kräfte" Das bedeutet, daß durch das Zusammengehen mehrerer Unternehmen in einem Netzwerk nicht automatisch ein Unternehmen seine gesamten Kapazitäten in das Netzwerk einbringt/einbringen muß. Für kleine und mittlere Unternehmen ist eine häufig praktizierte Variante derart, daß mit einem Teil der Beschäftigten feste Kunden weiterhin bedient werden, ein zweiter Personalanteil wirkt in Netzwerkeinheiten wie Bieter- und Realisierungsgemeinschaften - auch mehreren - je nach Erfordernis mit. Schon an dieser Stelle wird spätestens der beabsichtigte stabilisierende Effekt von Netzwerkaktivitäten für die beteiligten Firmen sichtbar.

- Ziel: „....auf die Realisierung von Wettbewerbsvorteilen zielende Organisationsform ökonomischer Aktivitäten..." - diese Beschreibung kann für die gegenwärtige Phase wie folgt präzisiert werden: Sicherung von Arbeitsplätzen!

- Die „Umschreibung der Beziehungen“ ist in der Praxis einfacher: es sind temporäre (also für einen bestimmten Zeitraum, für einen Auftrag gültige) Beziehungen zwischen eindeutig bestimmten, selbständigen Partnern, zumeist kleinen und mittleren Unternehmen

- Das Netzwerk reagiert branchenintern und/oder branchenübergreifend (letztere Variante ist für komplette Leistungen unumgänglich)

- Das Netzwerk kann regional, überregional oder interregional zusammengesetzt sein, ebenso kann es in den genannten Zonen oder zonenübergreifend arbeiten Netzwerke existieren in unterschiedlichen Bereichen der Gesellschaft, entscheidend für ihre Existenz ist eine eindeutige Zielstellung und das Bekenntnis der Netzwerkpartner zur Zusammenarbeit.

Entscheidend für die Benennung „Unternehmensnetzwerk" sei der zentrale Betrachtungsgegenstand im Netzwerk. Es geht um die Bündelung der Kräfte, der im Netzwerk beteiligten kleinen und mittleren Unternehmen, speziell werden die besonderen Fachkompetenzen für diese Bündelung verwendet. Es geht auch um die gemeinsame Leistungsplanung und -realisierung durch die Unternehmen im Netzwerk und es ist dazu die ausfuihrliche Kenntnis der Unternehmensdaten wie Branche, Beschäftigtenzahl und -qualifikation, Leistungen (Stärken und schwächen), angewendete Verfahren und Technologien, Erfahrungen, Umsatz, Marketing u.a. Unternehmensmerkmale erforderlich.

Dabei ist auch zu beachten, daß mit zunehmendem Netzwerkausbau, mit einer steigenden Funktionsintegration und der vielschichtigen Eindringung in die Marktebene, auch Einheiten wie Berater, Forscher, Verbände und andere wirtschaftliche, politische und ge- 
sellschaftliche Einheiten zum Zwecke der Gesamtlösung der Netzwerkaufgaben (nach innen und außen) in das Netzwerk integriert werden. Diese Einbindung von wichtigen, zumeist sogenannten „unproduktiven“ kommunikativen Leistungsträgern trägt aber nicht zur Begriffsveränderung des Netzwerkes bei sondern stabilisiert die Netzwerkfunktionen und fördert den Leistungseffekt. Durch die „Aufstockung“ der Netzwerkbasiseinheiten „Unternehmen“ und „Koordinierung“ mittels weiterer Funktionsträger erfolgt eine Verstärkung und Erweiterung der Netzwerkleistungen, dieser Prozeß ist sowohl leistungsfördernd als auch leistungssichernd zu werten, es sind auch marktsichernde und lobbyistische Aspekte erkennbar.

\section{Motivationspotentiale zur Netzwerk- bildung}

Die Motive zur Kooperation von kleinen und mittleren Unternehmen sind durch folgend qualitative und quantitative Faktoren gekennzeichnet.

Qualitativ unterschiedlichste Motive lassen sich durch die nachfolgenden Gründe, Überlegungen, Erfahrungen, Gegebenheiten und Situationen beschreiben:

- Der Konkurrenzkampf zwischen den Unternehmen nimmt auf regionaler, uiberregionaler und interregionaler Ebene zu

- Die Anzahl der Aufträge sowie deren Wertumfang sind im verarbeitenden wie auch im Bauhauptgewerbe (also auch im Baunebengewerbe) rückläufig

- Investoren bringen die ausführenden Firmen aus ihren Heimatregionen mit oder verwenden Billigarbeitskräfte aus nord-, ost- oder suideuropäischen Regionen, d.h., die vor Ort vorhandenen Fach- und Hilfskräfte bleiben weitestgehend unberüicksichtigt

- Investoren bringen die ausführenden Firmen mit, da diese Unternehmen schon mehrmals derartige Anlagen aufgebaut haben und damit iiber Erfahrungen verfuigen (es sind zum einen die fachlichen Erfahrungen und zum anderen auch die Erfahrungen im Umgang zwischen dem Investor und der realisierenden Firma)

- Großaufträge, wie sie z.B. im Chemiedreieck LeipzigHalle-Merseburg anfallen, eignen sich für allein arbeitende kleine Firmen nicht, häufig sind auch mittelständische Unternehmen personalseitig zu klein. Fazit: fuir die Hauptanlagenteile werden Großunternehmen verpflichtet, die ihrerseits für die „Restaufgaben“ kleine und mittlere Unternehmen zu häufig schwierigen Bedingungen binden

- Die von Großunternehmen ausgegebenen Präqualifikationsunterlagen für die Auftragsbewerbung und die damit verbundene Eignungskontrolle konfrontieren das sich bewerbenden Unternehmen mit den Anforderungen des lnvestors kann motivierende Lernprozesse auslösen

- Vom Auftraggeber werden komplette Leistungen gefordert, die ein Unternehmen nicht erbringen kann, es ergibt sich die Möglichkeit, in einem Netzwerk mittels partnerschaftlicher Beziehungen derartige Aufträge zu bearbeiten
- Der wiederholte Einsatz von vorrangig kleinen Unternehmen zu Leistungsbedingungen ohne nennenswerten Gewinn führt ebenfalls zu Überlegungen für den Zusammenschluß in Netzwerken

- Auch der hohe ausländische Leiharbeitnehmeranteil veranlaßt deutsche Firmen zum Nachdenken über neue Strukturen des Zusammengehens für definierte Aufträge

- Die ausgeschriebenen Themen überfordern häufig kleine aber auch mittlere Unternehmen bezuiglich der Personalgröße, der notwendigen Art und Anzahl von Ausrüstungen, der verfahrenstechnischen und technisch-technologischen Kapazitäten

- Kleine aber auch mittlere Einzelfirmen verfügen häufig nicht über die notwendigen finanziellen Mittel, um Material und Löhne für einen Auftrag vorzufinanzieren, ein Netzwerk hat verschiedene Möglichkeiten, das Problem zu lösen (ein insgesamt schwieriges Problem)

- Zu den Motivationspotentialen gehört auch der Wunsch, die Arbeitsplätze der eigenen Firma zu sichern, der Wunsch nach einer höheren und kontinuierlicheren Auslastung hochwertiger Ausrüstungen, der Wunsch zum Technologietransfer mit der Absicht der technologischen Aufrüstung des eigenen Unternehmens, der Wunsch zum Erfahrungsaustausch mit anderen Fachleuten der Region usw.

- Motivierend wirkt auch die Möglichkeit, die Forschungsleistungen und -ergebnisse unmittelbar durch die enge Bindung der Partner und die Vertrauensbasis im Netzwerk schneller als sonst umzusetzen, zu korrigieren, zu erproben - mit Nutzen in Leistungen für die Kunden umzuwandeln

- Netzwerkleistungen können aufgrund der nach innen offenen Angebotserarbeitung und der sinnvollen Auswahl von Netzwerkpartnern bezogen auf den jeweiligen Auftrag preisgünstiger sein als die Leistungen von allein agierenden Firmen

- Die Variabilität und Flexibilität in Netzwerken erhöht sich ohne unzumutbar hohen Aufwand des einzelnen Unternehmens allein schon durch das Vorhandensein eines größeren Mitarbeiterpotentiales. So kann man z.B. in der entscheidenden Phase der Auftragsrealisierung plötzlichen - immer wieder auftretenden „Auftragsstößen“ mit eigenen Ausrüstungen und dem eigenen Personal erfolgreich begegnen (die erforderliche Reserven befinden sich im Netzwerk)

- In einem größeren Personalpool sind im allgemeinen mehr Kenntnisse, Erfahrungen aber auch bessere Möglichkeiten für eine kostengünstige Qualifizierung vorhanden

- Das Zusammengehen von mehreren Firmen ermöglicht umfangreichere Aktivitäten der regionalen und überregionalen Werbung.

- Schließlich sind es auch wirtschaftlich, gesellschaftlich und politisch besonders engagierte Personen und Organisationen, die die Leistungen eines Unternehmens oder eines Netzwerkes würdigen oder bekanntmachen.

Quantitativ zeigt sich auf der Grundlage einer isw-Befragung [1] über die Bereitschaft zur Mitwirkung in Netzwerken folgendes Bild: 
- $31 \%$ der Befragten sind zur Mitarbeit bereit

- $44 \%$ der Befragten wären eventuell bereit

- $15 \%$ wollen nicht mitarbeiten und

- $10 \%$ äußerten sich nicht.

Überdenkt man diese Zahlen und berücksichtigt dabei z. B. die Auftragsentwicklung im Bauhauptgewerbe (Quelle: Jahreswirtschaftsbericht der Bundesregierung 96 '[5]) hinzu, so ist das Vertrautmachen mit alternativen Unternehmenskonzepten für kleine und mittlere Unternehmen ein existenzerhaltendes Thema höchster Priorität.

\section{Systematisierung, Funktions- und Funk- tionsträgerbestimmung in Netzwerken}

\subsection{Das System Netzwerk}

Netzwerke sind Systeme, die aus Teilsystemen bestehen und mittels deren (Teilsystem-) Funktionen und der zwischen diesen Teilsystemen bestehenden Kopplungen (materiell, informell, personell) Aufgaben erfuillen.

Netzwerke sind Systeme, die sich für eine zweckmäßige Betrachtung in Teilsysteme und Elemente zerlegen lassen. Bei Vahlens [6] heißt es zum Begriff „System“:

„System - abgegrenzte, geordnete Menge von sich gegenseitig beeinflussenden Elementen. Die zwischen diesen bestehende Ordnung wird als Struktur bezeichnet. Den Elementen kommen bestimmte Eigenschaften zu. Sie sind die kleinsten, nicht weiter zerlegbaren Bestandteile eines Systems.

(1) Ein System läßt sich in Teilsysteme zerlegen. Verfügt es neben internen Interaktionsbeziehungen auch über solche zu einem umgebenden System, so heißt es offenes, andernfalls geschlossenes System.

(2) Teilsysteme lassen sich zu Gesamtsystemen zusammenfassen.

(3) Dynamische Systeme verändern im Zeitablauf ihre Struktur bzw. die Elemente verändern aufgrund von Umwelteinflüssen ihre Eigenschaften. Im Gegensatz hierzu behalten statische Systeme auch bei Störungen ihre Struktur bei."

Um die Teilsysteme und ihr Zusammenwirken bestimmen und gestalten zu können, erscheint es sinnvoll, die Teilsysteme und die zwischen diesen ablaufenden Relationen zu betrachten. D.h., Unternehmensnetzwerke

- sind Systeme, die aus Teilsystemen bestehen, dies können Unternehmen, Teile von Unternehmen, Teilunternehmen aber auch mehrere Unternehmen sein

- diese Teilsysteme wiederum bestehen aus Elementen wie z.B. Equipments (gebäude- und ausrüstungsseitig), Material, Informationen und Personal

- realisieren im Sinne der Funktionserfuillung - mittels der Kopplung der Elemente und Teilsysteme zu Systemen - die Aufgabe(n)

- die zwischen den Elementen und Teilsystemen liegenden Relationen im Sinne von Kopplungen haben materiellen, informellen und personellen Charakter und realisieren die eigentliche Vernetzung
- aus der Art, Menge und dem Zeitverhalten dieser Relationen sowie der Lage der Teilsysteme und Elemente als Quell- und Zielpunkte dieser Relationen ist die Struktur des Systems (des Netzwerkes) bestimmbar; unter Hinzuziehung der Aufgaben und Zielstellung des Systems kann man auf offene, vernetzte Systeme schließen.

\subsection{Die Netzwerkebenen}

Netzwerke realisieren Aufgaben mittels der zur Lösung dieser Aufgaben notwendigen und installierten Funktionen, die durch Funktionsträger unterschiedlichster Art, deren Funktion und Leistungsfähigkeit und unter Zuhilfenahme der zwischen diesen Funktionsträgern existierenden Beziehungen.

Die Existenz der Funktionsträger sowie ihr Zusammenwirken mittels der genannten Relationen lassen sich analog eines ablauforganisatorischen Prozesses in Ebenen, sogenannten Netzwerkebenen darstellen.

Diese Netzwerkebenen sind Strukturbilder von Systemen und durch die in ihnen integrierten Funktionen bzw. Funktionsträger charakterisiert. Derartige Funktionsträger sind:

- Unternehmen als Basiseinheiten von Netzwerken

- Der Begriff Unternehmen reicht im Rahmen von Unternehmensnetzwerken vom produzierenden über das vertreibende Unternehmen bis hin zum Planungsund Beratungsunternehmen, dem Rechtsanwaltbüro und weiter zum Materiallieferanten bis hin zum Dienstleistungsbetrieb

- Der Begriff Unternehmen beginnt beim Handwerksbetrieb in Familiengröße und spannt sich bis zum mittelständischen Unternehmen mit mehreren hundert Beschäftigten

- Im Netzwerk sind außer diesen Basiseinheiten „Unternehmen“ weitere Funktionsträger als Struktureinheiten verschiedenster Leistungen an- oder eingegliedert. Herausragend $\mathrm{zu}$ benennen ist der Funktionsträger für die Aufgaben/Funktionen der Leitung und Koordinierung des Netzwerkes. Diese Aufgaben übernimmt im Netzwerk entweder der stärkste Betrieb/das für diesen Auftrag führende Unternehmen oder eine Koordinierungseinheit, deren Aufgaben erheblich über die des stärksten Unternehmens hinausgehen.

- Desweiteren gehören zu den Netzwerkeinheiten Architektur- und Planungsbüros, Technologietransferbüros, bei Förderprojekten der Projektträger und die wissenschaftliche Begleitung, Rechts- und Finanzberatungseinheiten, Forschungsteams an Universitäten, Fachhochschulen und Instituten, Marketingberater, regionale, über- und interregionale Kontakteinheiten sowie Kammern, Verbände und Gewerkschaften zuzüglich von Einrichtungen der Aus- und Weiterbildung.

- Die Erfahrungen zeigen, daß der Koordinierungsfunktion eine entscheidende Rolle zukommt. Es ist die Einheit aus Unternehmensleistungen und Netzwerkkoordinierung, die Voraussetzung für ein funktionierendes Netzwerk ist. 
Nachfolgend wird, auf der Grundlage der bisherigen Ergebnisse und Erfahrungen der wissenschaftlichen Begleitforschung, ein erster Versuch einer Ebenenordnung für Netzwerke unternommen. Zugrundegelgt werden die in das Netzwerk (die jeweilige Netzwerkebene) eingebundenen Funktionen bzw. Funktionsträger. Es werden vier Ebenen definiert.

\section{Netzwerkebene 1: Unternehmensgemeinschaft}

Die Netzwerkebene 1 besteht aus den Unternehmen, die die Netzwerkhauptfunktionen (Produkt herstellen, Erzeugnisse produzieren und vertreiben, Leistungen auch Dienstleistungen anbieten, ausfuihren/realisieren, verkaufen) erfüllen.

\section{Netzwerkebene 2: Bietergemeinschaft}

Unternehmen der Netzwerkebene 1 haben sich unter Führung der Koordinierungseinheit zu stabilen Arbeitseinheiten -Bietergemeinschaften- entwickelt.

\section{Netzwerkebene 3: Realisierungsgemeinschaft}

Die in der Netzwerkebene 2 integrierten Arbeitseinheiten werden bei Zuschlagserteilung als Realisierungsgemeinschaften tätig. Zur Erbringung kompletter Leistungen ist die Einbeziehung weiterer Funktionsträger notwendig, wie z.B. Forschungsdienstleister (Universitäten, Forschungsinstitute), Architektur- und Planungsbüros, Technologietransfereinrichtungen, Leistungen von Fachhochschulen, Marketing- und Finanzberater.

\section{Netzwerkebene 4: Integrationsgemeinschaft}

Zu den Funktionsträgern der Netzwerkebene 3 des Netzwerkes, die unmittelbar mit der Leistungserbringung oder der Koordinierung und Beratung beschäftigt sind treten Funktionsträger mit netzwerkstützender Funktion hinzu wie z.B. Verbände, Kammern und Gewerkschaften, kommunale, regionale, überregionale und interregionale Kontakteinheiten und dergleichen mehr.

Weiterführende Untersuchungen zu den Aufgaben und Inhalten der Netzwerkeinheiten in den genannten 4 Ebenen liegen bei den Autoren vor.

Die nachfolgenden Abbildungen 1 bis 4 zeigen die Strukturlayouts der Netzwerkebenen $1-4$.

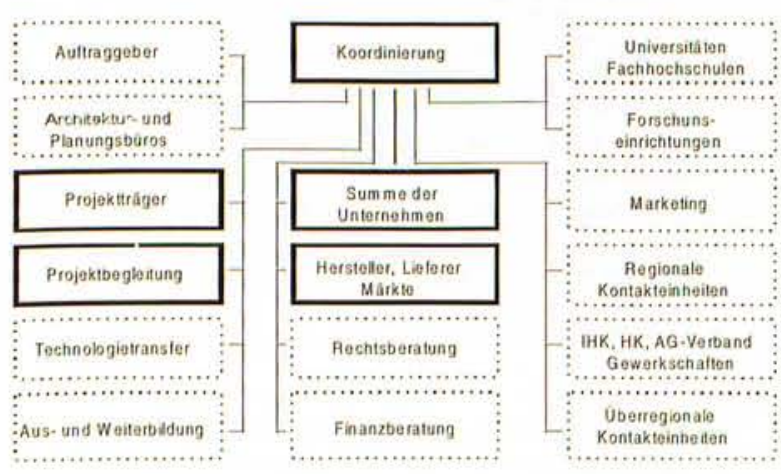

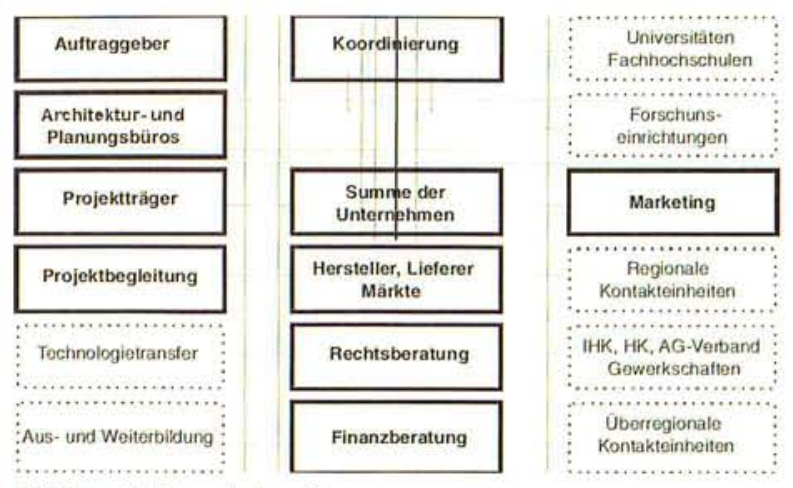

Abbildung 2: Netzwerkebene 2
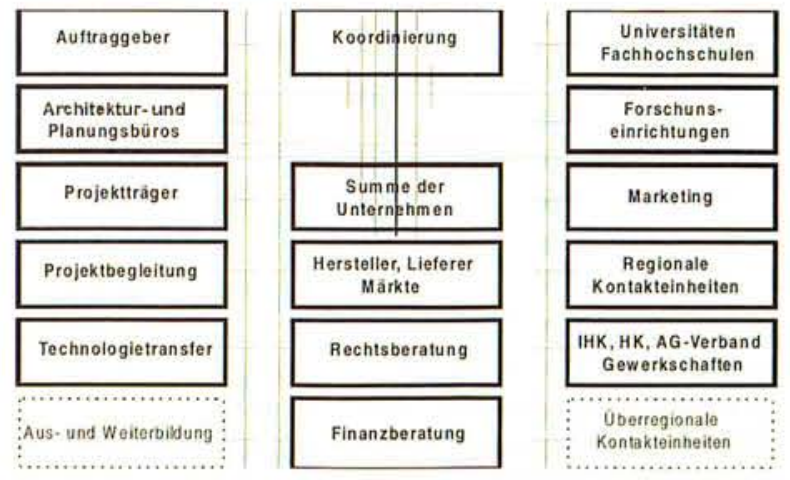

Abbildung 3: Netzwerkebene 3
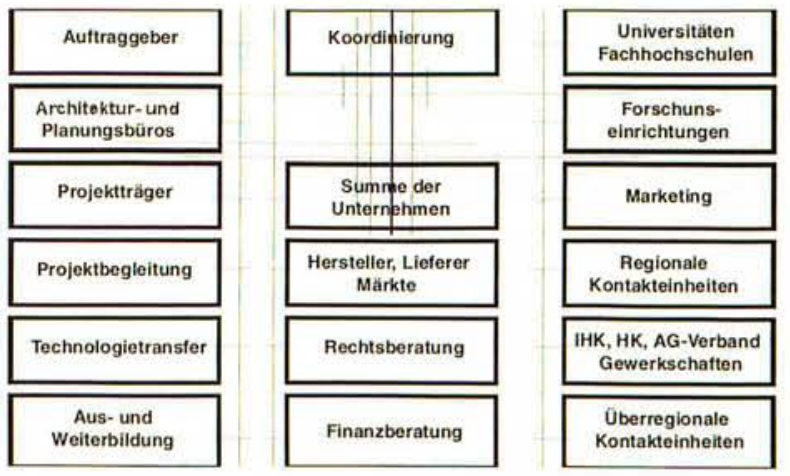

Abbildung 4: Netzwerkebene 4

\subsection{Funktionen und Funktionsträger im Netzwerk}

\subsubsection{Die Funktionen im Netzwerk}

Der Aufgabenlösungsprozeß und die abgeleiteten Funktionen des Netzwerkes wird von unterschiedlichsten Problemfeldern überlagert und es gilt uneingeschränkt, wenn Schädlich und Abicht in [1] schreiben: „Bei der Entstehung und dem Aufbau von Netzwerken kommt - trotz rationaler Zielsetzung der Unternehmen - der sozialen Komponente eine wesentliche Rolle zu. Netzwerkbeziehungen basieren auf Vertrauen, Gegenseitigkeit und kooperativem Verhalten. Ihre Aufnahme beruht auf Freiwilligkeit und ist zunächst nicht auf Dauer ausgerichtet. Wenn sich die Zusammenarbeit der Partner in einem Projekt bewährt hat, werden die Beziehungen aufrecht erhalten und intensiviert bzw. im Bedarfsfall wieder in Anspruch genommen."

Die Funktionen eines Netzwerkes, abgeleitet aus den Aufgaben des Netzwerkes, umfassen die Teilfunktionsfelder - Auftragsbeschaffung

- Angebotserarbeitung

- Leistungsrealisierung und

- Auftragsabschluß. 
Diese zugeordneten funktionellen Einheiten im Sinne von Teilsystemfunktionen sind - z.B. bezogen auf Netzwerke der Kategorie ökologisch-ökonomisches Bauen wie folgt zu benennen:

- Realisierung materieller und ideeller Leistungen

- Verkauf von Produkten/Erzeugnissen und Leistungen

- Marketingleistungen

- Leistungs-/Entwicklungs-/Erzeugnis-/Produktfinanzierung oder -vorfinanzierung

- Projektträgerschaft

- Beratung zu juristischen Problemen, z.B. Abfassung von Kooperationsverträgen

- Lieferleistungen/Einkauf

- Wissenschaftliche Projektbegleitung, -beratung

- Forschung und Entwicklung, Innovation

- Technologietransfer

- Bedingungen, Gegebenheiten und Forderungen der Städte und Gemeinden, kooperative Zusammenarbeit

- Kontakte, Forderungen, Mitwirkungen durch Bund und Länder

- Mitwirkungen aller Art durch die Kammern und Verbände sowie die Gewerkschaften

- Einbindung der potentiellen Auftraggeber.

Auf der Grundlage der Netzwerkaufgabenstellung und der genannten Teilsystemfunktionen können die Funktionsträger, d.h. die für die Umsetzung der Aufgabenstellung erforderlichen Unternehmen, Forschungs- und Planungseinrichtungen sowie weitere Netzwerkpartner ausgewählt bzw. bestimmt werden.

Die nachfolgende Abbildung 5 zeigt die Zuordnung der Teilsystemfunktionen zu den Ebenen im System Netzwerk.

\begin{tabular}{|c|c|c|c|c|c|}
\hline $\begin{array}{l}\text { Lffd. } \\
\text { Nr. }\end{array}$ & Teilsystemfunktion & $\begin{array}{c}\text { Ebene } \\
1\end{array}$ & $\begin{array}{c}\text { Ebene } \\
2\end{array}$ & $\begin{array}{c}\text { Ebene } \\
3\end{array}$ & $\begin{array}{c}\text { Ebene } \\
4\end{array}$ \\
\hline 1 & $\begin{array}{l}\text { Produzieren, Vernetzen, } \\
\text { Anbieten, Verkaufen }\end{array}$ & 1 & 1 & 1 & 1 \\
\hline 2 & Herstellen, Liefern, verkaufen & $0 / 1$ & $0 / 1$ & 1 & 1 \\
\hline 3 & Planen, Entwerfen, Gestalten & $0 / 1$ & 1 & 1 & 1 \\
\hline 4 & $\begin{array}{l}\text { Grundlagen- und angewandte } \\
\text { Forschung, innovativ fördern }\end{array}$ & 0 & $0 / 1$ & 1 & 1 \\
\hline 5 & $\begin{array}{l}\text { Projekt/Netzwerk fuihren, } \\
\text { Koordinieren, Controlling, } \\
\text { Kommunikativ fördern }\end{array}$ & 1 & 1 & 1 & 1 \\
\hline 6 & $\begin{array}{l}\text { Initiieren, Wissenschaftlich } \\
\text { begleiten, Mental fördern }\end{array}$ & $0 / 1$ & $0 / 1$ & $0 / 1$ & $0 / 1$ \\
\hline 7 & $\begin{array}{l}\text { Betriebswirtschaftlich beraten, } \\
\text { Finanziell begleiten, Fördern }\end{array}$ & 0 & 1 & 1 & 1 \\
\hline 8 & Vertragsrechtlich beraten & $0 / 1$ & 1 & 1 & 1 \\
\hline 9 & $\begin{array}{l}\text { Technisch informieren, } \\
\text { Kommunizieren, Kontaktieren }\end{array}$ & 0 & $0 / 1$ & 1 & 1 \\
\hline 10 & $\begin{array}{c}\text { Kommunizieren, Informieren: } \\
\text { regional }\end{array}$ & 0 & 1 & 1 & 1 \\
\hline 11 & $\begin{array}{c}\text { Kommunizieren, Informieren: } \\
\text { überregional }\end{array}$ & 0 & $0 / 1$ & $0 / 1$ & 1 \\
\hline 12 & $\begin{array}{l}\text { Aus- und Weiterbilden, } \\
\text { Qualifizieren }\end{array}$ & 0 & $0 / 1$ & $0 / 1$ & 1 \\
\hline 13 & $\begin{array}{l}\text { Kontaktieren, Beraten, } \\
\text { Betreuen, Unterstïtzen }\end{array}$ & $0 / 1$ & $0 / 1$ & 1 & 1 \\
\hline
\end{tabular}

Legende: 1 vorhanden 0 nicht vorhanden $0 / 1: 0$ oder 1 möglich

\subsubsection{Die Funktionsträger und ihre Zuordnung zu den} Netzwerkebenen

Die im Pkt. 3.3.1. genannten Funktionen sind im Netzwerk durch Funktionsträger zu realisieren. So z.B. das Entwerfen durch den Architekten, das Herstellen (Fertigen) und Montieren durch die Hersteller- und Montagebetriebe, das Beraten je nach Fachgebiet durch Rechtsanwälte oder Kreditinstitute, das Koordinieren von der Akquisition uiber die Auftragseinwerbung bis zu organisatorischen Fragen im Rahmen der Bieter- und Realisierungsnetzwerke durch das Koordinierungszentrum.

Zur Umsetzung der genannten Teilsystemfunktionen werden z.B. in einem Netzwerk zum ökologisch-ökonomischen Bauen die nachfolgenden Partner im Sinne von Funktionsträgern zusammengeführt:

- Baufirmen, die ihre Produktion in Richtung eines industriemäßigen Bauablaufes mit einem hohen Grad der Vorfertigung entwickeln wollen,

- Firmen aus dem Baunebengewerbe (Sanitär, Heizung, Lüftung, Elektro),

- Hersteller von Wandelementen, Fenstern, Dachelementen,

- Hersteller von Baustoffen und sonstigen Materialien, insbesondere auch aus der chemischen Industrie der Region Bitterfeld (Dämmstoffe, wie z. B. PUR-Hartschäume) oder auch Hersteller von Wärmeschutzverglasungen,

- Hersteller von Ausrüstungskomponenten (Sanitär, Elektro, Heizung, Solartechnik, Wärmespeichersysteme, Steuerungstechnik),

- Forschungseinrichtungen, insbesondere Bauphysiker, Baustofflabore,

- Architekten sowie

- Bildungseinrichtungen, die die Qualifizierung der Mitarbeiter übernehmen.

Die hinter dem Sammelbegriff „Funktionsträger“ oder auch „Partner“ agierenden Unternehmen vereint das Netzwerk durch gemeinsame Zielstellung, Aufgaben und deren Realisierung, sie handeln wie ein Unternehmen. Die nachfolgende Abbildung 6 zeigt die Zuordnung der Funktionsträger zu den Netzwerkebenen.

\begin{tabular}{|c|c|c|c|c|c|c|}
\hline $\begin{array}{c}\text { Lfd. } \\
\mathrm{Nr} .\end{array}$ & Funktionsträger & $\begin{array}{c}\text { Ebene } \\
1\end{array}$ & $\begin{array}{c}\text { Ebene } \\
2\end{array}$ & $\begin{array}{c}\text { Ebene } \\
3\end{array}$ & $\begin{array}{c}\text { Ebene Kennz. } \\
4\end{array}$ & \\
\hline 1 & Unternehmen & 1 & 1 & 1 & 1 & $\mathrm{~A}$ \\
\hline 2 & Hersteller/Lieferer & $0 / 1$ & $0 / 1$ & 1 & 1 & $\mathrm{~B}$ \\
\hline 3 & Planung/Entwurf & $0 / 1$ & 1 & 1 & 1 & $\mathrm{~A} / \mathrm{B}$ \\
\hline 4 & UNI, FH, Institute & 0 & $0 / 1$ & 1 & 1 & $\mathrm{C}$ \\
\hline 5 & $\begin{array}{c}\text { Projektträger: } \\
\text { Koordinierung }\end{array}$ & 1 & 1 & 1 & 1 & $\mathrm{~A}$ \\
\hline 6 & $\begin{array}{c}\text { Wissenschaftliche } \\
\text { Projektbegleitung }\end{array}$ & $0 / 1$ & $0 / 1$ & $0 / 1$ & $0 / 1$ & $\mathrm{~B}$ \\
\hline 7 & Kreditinstitut & 0 & 1 & 1 & 1 & $\mathrm{~B}$ \\
\hline 8 & Rechtsberater & $0 / 1$ & 1 & 1 & 1 & $\mathrm{~B}$ \\
\hline 9 & Technologietransfer & 0 & $0 / 1$ & 1 & 1 & $\mathrm{~B} / \mathrm{C}$ \\
\hline 10 & Kontakteinheiten regional & 0 & 1 & 1 & 1 & $\mathrm{~B}$ \\
\hline 11 & $\begin{array}{c}\text { Kontakteinheiten, } \\
\text { uber- und interregional }\end{array}$ & 0 & $0 / 1$ & $0 / 1$ & 1 & $\mathrm{~B} / \mathrm{C}$ \\
\hline 12 & Aus- und Weiterbildung & 0 & $0 / 1$ & $0 / 1$ & 1 & $\mathrm{C}$ \\
\hline 13 & $\begin{array}{c}\text { Kammern/Verbände } \\
\text { Gewerkschaften }\end{array}$ & $0 / 1$ & $0 / 1$ & 1 & 1 & $\mathrm{C}$ \\
\hline
\end{tabular}

\footnotetext{
Legende: 1 vorhanden 0 nicht vorhanden / möglich A: Basiseinheiten B: funktionsbedingt integriert C: einbezogen (erweiterte Integration)
} 


\section{Erfahrungen aus der bisherigen Netz- werkarbeit}

Die der Veröffentlichung zugrunde liegenden Pilotprojekte für KMU-Netzwerke bearbeiten nach einer erfolgreichen Planungs und -aufbauphase zur Zeit erfolgreich Aufträge als Bieter- und Realisierungsgemeinschaften.

Die nachfolgend dargestellten Erfahrungen sind aus der unmittelbaren persönliche Teilnahmen an vielen Aktivitäten, aus Gesprächen mit Führungskräften von KMUNetzwerken sowie ausschreibender Unternehmen abgeleitet. Sie sind ein Versuch, erste Ergebnisse einer beratenden Begleitung und permanenten Evaluierung des Entwicklungsprozesses vorzustellen.

\section{Initiierung und Konstituierung eines Netzwerks}

Das herausragende Argument zur Initiierung von KMUNetzwerken ist die reale Chance, daß kleine Unternehmen gleicher und verschiedener Branchen durch wechselseitige Leistungsergänzungen plötzlich gemeinsam den Anforderungen eines großen Auftraggebers gewachsen sind.

Die echte Anfangsbereitschaft zur Kooperation und der Wunsch, den potentiellen Partner relativ schnell kennenzulernen, war zwischen den hier betrachteten Unternehmen fast durchgängig vorhanden. Trotzdem war auffällig, daß es meist eines kräftigen Anfangsimpulses bedurfte, um Firmen erstmals zusammen zu bringen. Es scheint, als ob die gegenwärtige schwierige Wirtschaftslage (bzw. das freie Spiel der Marktkräfte) in Ostdeutschland allein nicht ausreicht, um eine sinnvolle KMU-Vernetzung zu initiieren.

Die wichtigsten Phasen bei der Anbahnung von Kooperationsbeziehungen kleiner und mittlerer Unternehmen sind das Kennenlernen, das offene Gespräch über mögliche gemeinsame Leistungen, das ehrliche Ansprechen möglicher Probleme, die gemeinsame Erarbeitung von Angeboten und die Offenlegung bzw. Koordination von Kalkulationen und Preisdifferenzen.

Die Kooperation von Handwerksfirmen ohne Regelungen zur juristischen Person des Verbundes ist im Bereich von großen Aufträgen auf Dauer unrealistisch. Der Auftraggeber erwartet die Leistungen aus einer Hand, er will nur mit einem Partner Verträge schließen. Außerdem müssen meist finanziellen Vorleistungen (Material-, Personal- und sonstige Kosten) zuverlässig zwischen den Netzwerkpartnern geteilt und Haftungsregeln vereinbart werden. Andernfalls sind auch die Banken nicht bereit, zum Beispiel Kreditrisiken einzugehen.

Die vertragsrechtlichen Beziehungen zwischen Netzwerkunternehmen sind deshalb mit großer Umsicht zu gestalten. Die häufig praktizierte Form einer Arbeitsgemeinschaft (ARGE) ist nicht als generelles Muster anwendbar. Es müssen bestimmte Voraussetzungen und Forderungen zu ihrer Anwendung erfuillt sein. Ein Problem, besonders bei extrem unterschiedlichen Leistungsträgern, sind Bürgschaftsfragen.

KMU-Netzwerke müssen zur Sicherung ihrer Existenz am Markt wie Großunternehmen ein Image aufbauen, ihren Bekanntheitsgrad entwickeln und sich auch eine entsprechende Lobby schaffen. Dafür sind neue (für die beteiligten KMU oft ungewohnte) Leistungen aus dem Verbund heraus arbeitsteilig zu entwickeln und finanziell zu gewährleisten (Netzwerk- und Unternehmenspräsentation, Werbeblätter, Leistungskataloge usw.) Als besonders erfolgreich haben sich regionale Informationsveranstaltungen (Workshops) erwiesen, in denen die Netzwerk-Partner mit kompetenten und interessierten Vertretern aus Wirtschaft (insbesondere auch potentielle Großauftraggeber), Politik und Wissenschaft zusammentreffen. Hier besteht z.B. die Möglichkeit, vorausschauend Geschäfte anzubahnen und um Bekanntheit und Unterstiitzung zu werben.

\section{Akquisition von Aufträgen}

Fehlendes Image und überzeugende Referenzobjekte, fehlende Zertifizierung, mangelnde Erfahrungen im Projektmanagement, geringe Personalkapazität, „schmale“ Leistungsangebote sowie mangelnde Finanzkraft erschweren generell die Auftragsbeschaffung für KMU Zunehmend werden in Ausschreibungen komplexe Leistungen ,aus einer Hand“ erwartet, die nur schwer oder gar nicht von kleine Unternehmen, wie z.B. Handwerksfirmen erbracht werden können.

Viele kleine und auch mittlere Unternehmen haben aber nur wenige Erfahrungen in der zeitaufwendigen Beschaffung und Sichtung von Ausschreibungsunterlagen. Außerdem wirken negativ Erfahrungen der Führungskräfte aus dem Umgang mit öffentlichen Ausschreibungen als psychologische Barriere. Schließlich belastet auch die zusätzliche Konkurrenz von Großunternehmen, die mit ihren Angeboten zunehmend auch in typische KMU-Leistungsbereiche eindringen.

Oft wird noch unterschätzt, daß ein Angebot vom potentiellen Partner bereits als Referenzleistung angesehen wird (Termineinhaltung bzw. -unterbietung, Vollständigkeit, technische Perfektion usw.). Deshalb hat es sich für noch relativ unbekannte Bietergemeinschaften bzw. KMU-Netzwerke bewährt, bei der Abgabe von Angeboten ihren Bekanntheitsgrad durch Zusatzinformationen zum generellen Leistungsprofil nachhaltig zu verbessern. Dabei ist zu beachten, daß das im Netzwerk zu organisierende Beobachten, Auswählen, Einholen und Verteilen von Informationen uiber relevante Ausschreibungen ohne Nutzung moderner Informationsund Kommunikationstechnologien sehr zeitaufwendig ist.

\section{Führung und Organisation von Geschäftsprozessen}

Es zeigt sich zunehmend, daß durch partnerschaftliches Zusammenarbeiten mehrerer unterschiedlich großer Firmen, geführt von einem starken Unternehmen, die vom Auftraggeber geforderten, auch branchenübergreifenden Leistungen zuverlässig bereitgestellt können. Selbst eine für den Verbund eventuell notwendige Unterstiitzung durch die Hausbank (Beratung, Kredite, Bürgschaften) kann durch den glaubhaften Nachweis einer stabile Partnerschaft durchaus erleichtert werden. Zu den herausragenden Vorteilen von KMU-Netzwerken gehört die Kapazitätserhöhung und Kompetenzergän- 
zung. So entstand zum Beispiel in einem Verbund aus acht kleine Unternehmen der Elektrobranche ein Pool von etwa 160 Mitarbeitern, darunter Meister, ingenieurtechnisches Personal, elektrotechnische Fachkräfte, Meß- und Regelungstechniker sowie Facharbeiter mit Schweißerlaubnis. Damit werden neue Maßstäbe an die Führung dieses Verbundes gesetzt. Die außerdem damit verbundene Erhöhung von Flexibilität, Variabilität und Reaktionsvermögen bezogen auf Aufgabentreue und Termineinhaltung muß ebenfalls durch eine professionelle Führung und Organisation abgesichert werden.

Als nachteilig hat es sich erwiesen, daß das Netzwerk anfangs noch kein eigenen Namen, kaum Referenzen, nur wenige Kontakte vorweisen konnte. Demgegenüber verfügen Großunternehmen über langjährige Kontakte zu potentiellen Auftraggebern, man kennt sich und hat schon öfter zusammengearbeitet. Deshalb ist es dringend erforderlich, Netzwerke nicht nur als Bieter- und Realisierungsgemeinschaften sondern auch als Integrationsgemeinschaften zu gestalten, die mit ihrer Leistung auch ein überzeugendes Image entwickeln.

Die komplizierten Arbeitsfelder Marketing, Akquisition und Qualitätssicherung erhalten für größere Aufträge im Netzwerk eine neue Dimension. Ein jetzt zu realisierender Auftrag erhöht die bisher üblichen Leistungserwartungen und erzwingt im einzelnen Unternehmen nicht nur aus Kostengründen kooperatives Handeln auch bei der Entwicklung von Managementkompetenz. So werden kleine und mittlere Unternehmen zum Beispiel bei der Auftragsvergabe für Großprojekte trotz mehrfacher Beteuerung durch die politischen Ebenen nicht ausreichend beruicksichtigt.

Die Beteiligung an Bieter- und Realisierungsgemeinschaften fuihrt insgesamt zu einer ungewohnten Doppelbelastung. Als fairer Netzwerkpartner muß das mittelständischen Unternehmen zur Entwicklung und Stabilisierung des Kooperationsverbundes beitragen. Als juristisch selbständige Wirtschaftseinheit muß es aber auch weiterhin initiativreich am Markt agieren und ggf. auf identischen bzw. angrenzenden Geschäftsfeldern mit Partnern aus dem „eigenen“ Netzwerk konkurrieren. Auf die damit verbundenen neuen Herausforderungen sind die meisten KMU-Führungskräfte nicht vorbereitet.

Dazu ist netzwerkintern das generelle Prinzip der Gleichbehandlung aller beteiligten Unternehmen unter Beachtung der realen Bedingungen zu konkretisieren und von allen Beteiligten akzeptierte Verfahrensweisen als Regelwerk zu vereinbaren. So kann es zum Beispiel sinnvoll sein, auftragsabhängig jeweils neu festzulegen, welches Unternehmen das Auftragsmanagement übernimmt und welche Unternehmen mit einigen Mitarbeitern „nur“ zur Personalverstärkung beitragen. Das übergreifende Problem ist die neu zu entwickelnde Fähigkeit, mit der flexiblen Regelung notwendiger Entscheidungsprozesse auch die Vertrauensbildung im Netzwerk zu fördern (Netzwerkkoordinierung).

Infolge des außerordentlichen Kompliziertheits- und Neuheitsgrads der Kooperation in KMU-Netzwerken entsteht zumindest in der Anfangs- und Konsolidierungsphase ein erheblicher Betreuungs- und Beratungsbedarf. So fehlen zum Beispiel sehr häufig wichtige Kontakte nicht nur untereinander sondern auch zu
Liefereranten, Planungsbüros, Bauämtern sowie zu großen Unternehmen. Deshalb hat es sich im vorliegenden Fall bewährt, eine durch die Landesregierung geförderte Koordinierungseinheit in repräsentativen Pilotprojekten zeitlich befristet zur Führung, Anleitung und Unterstiutzung des Netzwerkes sowie in Gestalt des isw zur wissenschaftlichen Begleitung einzusetzen.

Die Erfahrungen zeigen, daß die Dauer einer öffentlich geförderten Netzwerkkoordinierung von der Marktstruktur und von der Lernfähigkeit der beteiligten KMU abhängt. Die Notwendigkeit einer äußeren Koordinierungsunterstützung entfällt, sobald grundsätzliche Managementfunktionen wie Marketing, Öffentlichkeitsarbeit, Kontaktpflege, Auftragsbeschaffung aber auch interne Koordinierungsaufgaben stabil aus dem Netzwerk heraus sichergestellt sind.

\section{Nutzung moderner Informations- und Kommunikati- onstechnologien}

Netzwerke sind mehr noch als Einzelunternehmen gefordert, eine große Menge von Informationen schnell zu erfassen und gezielt zu verteilen. Dieser zeit- und kostenintensive Prozeß erfordert den Einsatz moderner Informations- und Kommunikationstechnologien, um zunehmend mit Großunternehmen um Aufträge und Ansehen konkurrieren zu können.

Besonders am Beispiel der Reaktion der kleinen und mittleren Unternehmen im Netzwerk auf Ausschreibungen hat sich gezeigt, daß wegen mangelnder Erfahrungen im Umgang mit Informations- und Kommunikationstechnologien viel zu langsam agiert wird. Insbesondere wird noch zu wenig der „elektronische Kontakt“ zu Großunternehmen gesucht. Eine noch zu entwickelnde Fähigkeit betrifft das parallele Reagieren auf Ausschreibungen verschiedenster Art und das gleichzeitige Anbieten der Leistungen einer Bietergemeinschaft z.B. im Internet.

Außerdem mangelt es in den Netzwerken noch an der Fähigkeit, den Markt nach vorhandenen Leistungen und Leistungsträgern zu analysieren und zu selektieren, Marktluicken und Nischen zu erkennen und gezielt zu bearbeiten. Damit könnte die Marktpräsenz der Unternehmens- und Netzwerkeinheiten aber wesentlich verbessert werden (z.B. durch Beschaffung von Ausschreibungsinformationen der öffentlichen, beschränkten und freihändigen Vergabe mittels elektronischer Medien und privater Kontakte).

In der Planungs- und Aufbauphase sind wegen der mangelhaften Nutzung von Informations- und Kommunikationstechnologien große Zeitverluste bei der Gewinnung von Firmen, bei der Bekanntmachung des Netzwerkes, bei Kontakten zu Vertretern aus Verwaltungen, Kammern, Bildungs- und Forschungseinrichtungen, Planungseinheiten der Region usw. entstanden.

Es hat sich aber auch gezeigt, wie nützlich Informationsund Kommunikationstechnologien für die Detaillierung und Pflege von Geschäftsbeziehungen sind. Dazu gehören vor allem Informationen über Mitbewerber, über potentielle Auftraggeber, iiber positive und negative Gewohnheiten von Geschäftspartnern, über Insiderkreise u.ä. aber auch zum Erhalt und Ausbau von Forschungs- und Entwicklungskapazitäten. 


\section{Resümeé}

Die beiden Pilotnetzwerkprojekte befinden sich trotz unterschiedlicher Voraussetzungen, Aufgaben- und Zielstellung sowie besonderer regionaler Gegebenheiten in der Realisierungsphase, die durch ein umfangreiches Agieren der Koordinierungseinheiten sowie von Bieterund Realisierungsgemeinschaften charakterisiert ist. Schrittweise werden weitere, netzwerkstabilisierende Partner einbezogen, sodaß sich der Übergang zur Netzwerkebene 4 - Integrationsgemeinschaft - vollzieht.

\section{Literaturverzeichnis}

[1] Schädlich, M.; Abicht,L.; Dubiel, G.;Hande, W.; Schönfeld, P.:

Studie zum Thema „Netzwerke in Sachsen-Anhalt". Isw Halle-Leipzig, 1995.

[2] Schädlich, M.; Abicht, L.; Bärthel, H.:

„Aufbau eines Netzwerkes zur Verwirklichung von Systemlösungen des ökologisch-ökonomischen Bauens unter Beteiligung von KMU verschiedener Branchen“, Forschungsbericht I, Januar 1997. isw Halle-Leipzig

[3] Schädlich, M.; Abicht, L.; Bärthel, H.:

„Aufbau eines absatzorientierten Netzwerkes kleiner und mittlerer Unternehmen im Raum Leuna-Merseburg" isw Halle-Leipzig, Forschungsbericht I, Oktober 1996.

[4] Sydow, J.:

Strategische Netzwerke (Evolution und Organisation).

Wiesbaden, 1992.

[5] Vorrang für Beschäftigung/Jahreswirtschaftsbericht der Bundesregierung 96'.

Bundesministerium für Wirtschaft, 1996.

[6] Vahlens Großes Wirtschaftslexikon, Band 4 Deutscher Taschenbuch Verlag, München, 1987.

[7] Schräder, A.: Management virtueller Unternehmungen, Campus Verlag, Frankfurt/New York, 1996.

[8] Goldman, S.L., Nagel, R.N., Preiss, K., Warnecke, H.-J.: Agil im Wettbewerb. Die Strategien der virtuellen Organisation zum Nutzen des Kunden Springer-Verlag Berlin Heidelberg, 1996.

\section{Verfasser}

Dr.-Ing. Helmut Bärthel

Institut für Strukturpolitik und Wirtschaftsförderung Halle-Leipzig (isw)

Tel. 0345/51 70 704/05; Fax 0345/51 70 706;

ISDN 03455213 60/70

Prof. Dr. sc. oec. Michael Büttner

Technische Fachhochschule Wildau

Fachbereich Wirtschaft, Verwaltung und Recht

Tel. (03375) 508-970 oder 950 (auch Fax) 